\title{
Using Neural Imaging to Inform the Instruction of Mathematics
}

\author{
John Anderson \\ Department of Psychology \\ Carnegie Mellon University, USA \\ ja+@cmu.edu
}

I will describe research using fMRI to track the learning of mathematics with a computer-based algebra tutor. I will describe the methodological challenges in studying such a complex task and how we use cognitive models in the ACT-R architecture to interpret imaging data. I wll also describe how we can use the imaging data to identify mental states as the student is engaged in algebraic problems solving. 